\title{
Viral epidemiology of respiratory infections among children at a tertiary hospital in Southern Brazil
}

\author{
Fernanda de-Paris ${ }^{[1],[2]}$, Caroline Beck ${ }^{[3]}$, Márcia Rosane Pires ${ }^{[4]}$, Rodrigo Pires dos Santos ${ }^{[4]}$, \\ Ricardo de Souza Kuchenbecker ${ }^{[3]}$ and Afonso Luis Barth ${ }^{[1],[2]}$
}

[1]. Serviço de Patologia Clínica, Laboratório de Biologia Molecular, Unidade de Pesquisa Biomédica, Hospital de Clínicas de Porto Alegre, Universidade Federal do Rio Grande do Sul, Porto Alegre, RS. [2]. Programa de Pós-Graduação em Ciências Médicas, Faculdade de Medicina, Universidade Federal do Rio Grande do Sul, Porto Alegre, RS. [3]. Programa de Pós-Graduação em Epidemiologia, Instituto de Avaliação de Tecnologia em Saúde, Universidade Federal do Rio Grande do Sul, Porto Alegre, RS. [4]. Comissão de Controle de Infecção Hospitalar, Hospital de Clínicas de Porto Alegre, Universidade Federal do Rio Grande do Sul, Porto Alegre, RS.

\begin{abstract}
Introduction: This study reports the pediatric epidemiology of respiratory syncytial virus (RSV), influenza (IF), parainfluenza (PIV), and adenovirus (ADV) at Hospital de Clínicas de Porto Alegre. Methods: Cases of infection, hospitalizations in intensive care units (ICUs), nosocomial infections, and lethality rates were collected from 2007 to 2010. Results: RSV accounted for most nosocomial infections. Intensive care units admission rates for ADV and RSV infections were highest in 2007 and 2010. During 2008-2009, H1N1 and ADV had the highest ICU admission rates. ADV had the highest fatality rate during 2007-2009. Conclusions: Each virus exhibited distinct behavior, causing hospitalization, outbreaks, or lethality.
\end{abstract}

Keywords: Respiratory viruses. Pediatric patients. Influenza A (H1N1) virus.

Respiratory tract infections (RTIs) are a major cause of morbidity and mortality in children worldwide ${ }^{1}$. According to World Health Organization data, RTIs account for the deaths of approximately 4 million children per year. Two-thirds of these deaths occur in infants during the first year of life, and $90 \%$ occur in developing countries. Viruses are the most common causative agents of RTIs and are responsible for a substantial percentage of childhood mortality ${ }^{2}$. Traditionally, the most widely researched viruses implicated in RTIs include human respiratory syncytial virus (RSV), rhinovirus (RHV), influenza (IF), parainfluenza viruses (PIV), and adenovirus (ADV) ${ }^{3}$. These viruses circulate within the community and cause many hospitalizations. Viral respiratory nosocomial infections are also observed throughout the year. Increased hospitalization rates and nosocomial outbreaks can create a major financial burden ${ }^{4}$.

The importance of respiratory virus surveillance has grown over the last 10 years because of the unexpected emergence of several new respiratory viruses, including influenza A (H5N1), severe acute respiratory syndrome (SARS) coronavirus, coronavirus NL63, human bocavirus, and influenza A virus $(\mathrm{H} 1 \mathrm{~N} 1)^{5}$. Furthermore, the seasonality of some respiratory viruses is well established, and viral surveillance and laboratory-

Address to: Dria Fernanda de-Paris. Laboratório de Biologia Molecular/Unidade de Pesquisa Biomédica/HCPA. Rua Ramiro Barcelos 2350/2 ${ }^{\circ}$ andar, 90035-903 Porto Alegre, RS, Brasil.

Phone: 5551 3359-8860; Fax 5551 3359-8310.

e-mail: fparis@hcpa.ufrgs.br; fparis@terra.com.br

Received 23 April 2013

Accepted 27 August 2013 based diagnostics play an important role in guiding the timing of prophylaxis and other interventions. However, this seasonality may be variable. In temperate regions, for instance, viral infections tend to peak in the late fall and winter, whereas in tropical regions, they occur over extended periods associated with the rainy season ${ }^{6}$. In Brazil, a continental country that is host to many different climates, regional surveillance studies on viral patterns play an essential role in the planning of prophylaxis programs and other interventions. Thus, this study aimed to report the epidemiology of major respiratory viruses (RSV, IF, PIV, and ADV) among pediatric patients who were treated at Hospital de Clínicas de Porto Alegre (HCPA) between 2007 and 2010, including data on the 2009 influenza A(H1N1) pandemic.

Porto Alegre is the capital of the Brazilian State Rio Grande do $\mathrm{Sul}$, which is located at $10 \mathrm{~m}$ above mean sea level, latitude 30.01 $\mathrm{S}$, and longitude $51.13 \mathrm{~W}$. It has a mean annual temperature of $19^{\circ} \mathrm{C}$ and an area of $497 \mathrm{~km}^{2}$ with a population of approximately 1.5 million. Hospital de Clínicas de Porto Alegre is a universityaffiliated tertiary referral hospital with over 800 beds that serves the population of Porto Alegre and its metropolitan region. This study followed an observational design, and the data on all variables were collected from children (age 0-12 years) who were treated at HCPA between 2007 and 2010 (retrospective study). We obtained the number of cases of respiratory infection attributable to each viral agent of interest, the number of patients admitted to general wards and intensive care units (ICUs), the number of nosocomial infections, and lethality rates. The study protocol was approved by the HCPA Research Ethics Committee.

The laboratory detection of viruses (RSV, ADV, IF, and PIV) in nasopharyngeal aspirates was performed with an indirect immunofluorescence (IIF) assay using commercially 
available monoclonal antibodies (Medivax ${ }^{\circledR}$, Dublin, Ireland). Influenza $\mathrm{A}(\mathrm{H} 1 \mathrm{~N} 1)$ was detected using real-time polymerase chain reaction (PCR) as per the Centers for Disease Control and Prevention (CDC) protocol' 7 . This assay was performed in Brazilian reference laboratories.

The number of IIF-confirmed cases of infection with each respiratory virus of interest (RSV, IF, PIV, and ADV) was obtained from HCPA Infection Control Committee reports, as was the number of hospitalizations in general wards and ICUs. The criteria for nosocomial infection included the absence of respiratory symptoms at the time of admission and the detection of a respiratory virus within 7 days after admission ${ }^{8}$. Nosocomial infection rates were calculated as the percentage of nosocomial infections attributable to each virus divided by the total number of nosocomial infections attributable to all viruses x 100 .

The number of deaths due to viral respiratory tract infection was obtained, and the case lethality rates for each virus were calculated. Case lethality was calculated using the formula number of deaths/number of cases x 100. All results were obtained by descriptive analysis.

Approximately 12,160 pediatric patients were treated per year at the HCPA Emergency Department. The Emergency Department is the point of entry for the majority of patients treated at HCPA. The number of patients treated and the number of suspected and confirmed cases of viral RTIs (due to RSV, IF, PIV, and ADV) followed an upward trend during the winter months (June to August) of each year (Figure 1).

The number of confirmed cases stratified by virus and severity (admission to general wards or ICUs) is shown in Table 1. This table shows that RSV and ADV were responsible for the highest rates of ICU admission in 2007 and 2010, whereas H1N1 and ADV accounted for the highest rates of ICU admission in 2008 and 2009.

Table 2 shows that RSV was responsible for the highest rates of nosocomial infection throughout the study period $(81.5 \%, 61 \%, 37.7 \%$, and $53.5 \%$ in 2007 through 2010 , respectively).

The case fatality rates observed in 2007 through 2010 , respectively, were as follows: $\mathrm{ADV}=7.1 \%, 16.7 \%, 7.1 \%$, and $0 \%$; IF $=0 \%, 5 \%, 4.7 \%$, and $0 \%$; PIV $=0 \%, 1.5 \%, 0.9 \%$, and $0 \%$; and RSV $=1.5 \%, 1.4 \%, 1.5 \%$, and $2.3 \%$. In 2009 , the case fatality rate for $\mathrm{H} 1 \mathrm{~N} 1$ was $7.1 \%$. ADV had the highest case fatality rates. In 2009, the case fatality rate of H1N1 was similar to that of ADV.

In temperate regions, the rates of viral infection generally tend to increase in the late fall and winter, whereas in tropical regions, this increase occurs over extended periods associated with the rainy season ${ }^{6}$. In our study, conducted in Porto Alegre, we observed a trend toward increases in the number of confirmed cases of viral infection in the winter months (June to August) of each year (Figure 1). A substantial number of RSV and influenza cases occurred in these months. These viruses, particularly RSV, are highly prevalent among children (Table 1). Consequently, annual RSV outbreaks lead to increased presentation of children to the HCPA Emergency Department. The frequency and severity of RSV infections in this age group appear to be a consequence of complex and multifactorial events, mainly involving immunological factors ${ }^{9}$. Palivizumab, a humanized monoclonal antibody, is currently recommended as prophylaxis for certain patient groups at risk of severe RSV infection ${ }^{4}$.

TABLE 1 - Number of confirmed cases by virus, rate of admission to general wards and admission to intensive care unit.

\begin{tabular}{lrrrr}
\hline Human respiratory syncytial virus & 2007 & 2008 & 2009 & 2010 \\
\hline Human Adenovirus & & & & \\
N & 14 & 12 & 14 & 18 \\
A & 8 & 10 & 9 & 14 \\
$\%$ & 57.1 & 83.3 & 64.3 & 77.8 \\
ICU & 2 & 2 & 2 & 2 \\
$\%$ & 14.3 & 16.6 & 14.3 & 11.1 \\
\hline
\end{tabular}

Influenza virus

\begin{tabular}{lrrrr} 
N & 25 & 20 & 105 & 6 \\
$\mathrm{~A}$ & 5 & 11 & 50 & 4 \\
$\%$ & 20.0 & 55.0 & 47.6 & 66.7 \\
$\mathrm{ICU}$ & 2 & 4 & 5 & 0 \\
$\%$ & 8.0 & 20.0 & 4.8 & 0.0 \\
\hline
\end{tabular}

Influenza A (H1N1) virus

\begin{tabular}{llrrr}
$\mathrm{N}$ & - & - & 28 & - \\
$\mathrm{A}$ & - & - & 18 & - \\
$\%$ & - & - & 64.3 & - \\
$\mathrm{ICU}$ & - & & 7 & - \\
$\%$ & - & - & - \\
\hline
\end{tabular}

Parainfluenza viruses

\begin{tabular}{lrrrr} 
N & 68 & 136 & 105 & 98 \\
$\mathrm{~A}$ & 23 & 34 & 67 & 60 \\
$\%$ & 33.8 & 25 & 63.8 & 61.2 \\
$\mathrm{ICU}$ & 5 & 7 & 5 & 2 \\
$\%$ & 7.3 & 5.1 & 4.8 & 2.0 \\
\hline
\end{tabular}

Human respiratory syncytial virus

\begin{tabular}{lrrrr} 
N & 201 & 296 & 264 & 307 \\
$\mathrm{~A}$ & 119 & 168 & 157 & 259 \\
$\%$ & 59.2 & 56.7 & 59.5 & 84.4 \\
$\mathrm{ICU}$ & 32 & 38 & 25 & 33 \\
$\%$ & 15.9 & 12.8 & 9.5 & 10.7 \\
\hline
\end{tabular}

$\mathrm{N}$ : number of confirmed cases by each virus; A: number of cases with admission to general wards by each virus; ICU: number of cases with admission to intensive care unit. 

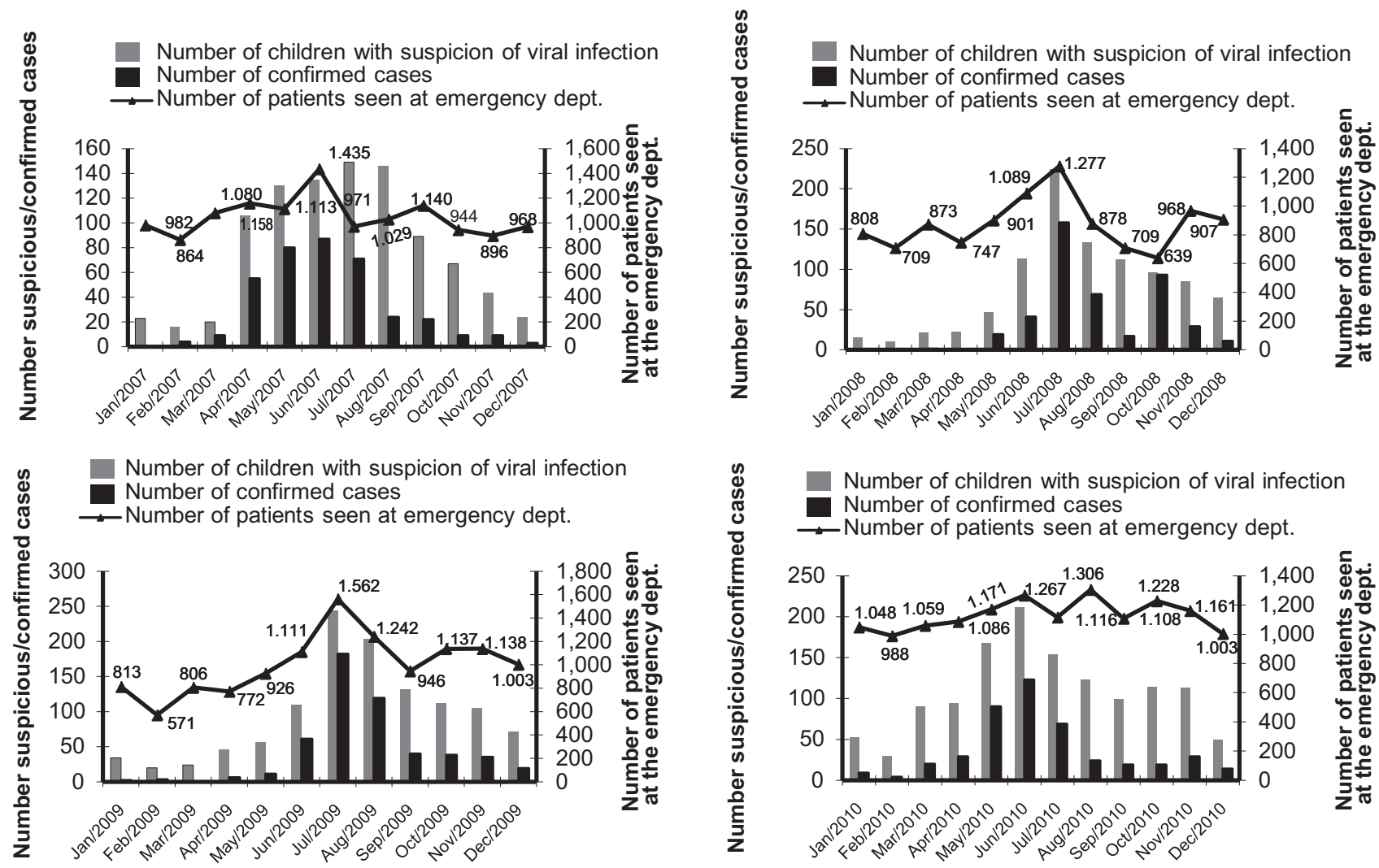

FIGURE 1 - Number of patients treated for suspected infection with respiratory virus and confirmed cases of infection, 2007-2010.

TABLE 2 - Rates of nosocomial infection by respiratory viruses (ADV, IF, H1N1, PIV and RSV) in each year (2007-2010).

\begin{tabular}{lccccc}
\hline & \multicolumn{5}{c}{ Nosocomial infections* } \\
\cline { 2 - 6 } Years & ADV (\%) & IF (\%) & H1N1 (\%) & PIV (\%) & RSV (\%) \\
\hline 2007 & 11.0 & 0.0 & 0.0 & 7.0 & 81.5 \\
2008 & 12.0 & 2.0 & 0.0 & 24.5 & 61.0 \\
2009 & 1.4 & 29.0 & 4.0 & 27.5 & 37.7 \\
2010 & 4.6 & 2.0 & 0.0 & 39.5 & 53.5 \\
\hline
\end{tabular}

ADV: human adenovirus; IF: influenza virus; H1N1: influenza A (H1N1) virus; PIV: parainfluenza viruses; RSV: human respiratory syncytial virus. *nosocomial infection rates were calculated as the percentage of nosocomial infections caused by each virus/total number of viral nosocomial infections x 100 .

Despite its proven efficacy in reducing hospitalizations attributable to RSV, palivizumab is still exceedingly expensive for developing countries such as Brazil. Therefore, reducing costs through the optimization of drug administration may allow more children to benefit from RSV prophylaxis ${ }^{10}$.

Concerning nosocomial infections, RSV is the foremost cause of hospital-acquired respiratory infections among children $^{8}$. The rates of hospital-acquired RSV infection observed in this study are consistent with this information (Table 2). However, these rates displayed some variation in each year of the study period, and a decrease occurred in 2009. This downward trend in 2009 may have been associated with the extensive circulation of H1N1 in this period. A similar phenomenon was reported by Mak and co-workers in Hong Kong and is most likely explained by virus interference ${ }^{11}$.

Regarding the severity of respiratory infections, using ICU admission as a surrogate measure, we observed higher rates of ICU admission for RSV and ADV infection in 2007 and 2010, whereas in 2008 and 2009, the highest rates of ICU admission were attributable to H1N1 and ADV (Table 1). Adenovirus infections often lead to severe clinical consequences in hospitalized patients $^{12}$, and these infections are a matter of great concern to the members of hospital infection control committees. During 2009, H1N1 accounted for a substantial portion of ICU admissions. Libster and colleagues also reported higher hospitalization rates due to H1N1 infection among Argentinean children. In Argentina, the rates of hospitalization for H1N1 were double those for seasonal influenza in $2008^{13}$. We observed a similar increase in hospitalization rates in our sample, demonstrating that the emergence of H1N1 had a major impact on our health system. However, RSV can account for even higher ICU admission rates, as observed in 2007 and 2010 in this study. These variations are most likely associated with circulating RSV genotypes and with the extent of the annual RSV outbreak in each year ${ }^{14}$. 
Our study also assessed the case lethality rates of viral RTIs. Mirroring the severity of respiratory infections as measured by ICU admission, ADV and H1N1 had the highest case fatality rates, as expected. Linch and coworkers reported that ADV disease is more severe, and dissemination more likely, in patients with impaired immunity ${ }^{12}$. Because of their severe clinical manifestations and ability to start outbreaks, ADV infections are kept under close surveillance in the hospital setting. As for H1N1, this new emergent strain of influenza displayed a distinct severity profile among pediatric patients. Whereas seasonal influenza had case lethality rates of approximately $5 \%$, the lethality rate of $\mathrm{H} 1 \mathrm{~N} 1$ was $7.1 \%$. Other studies have also noted that influenza A (H1N1) is associated with substantial pediatric mortality ${ }^{13,15}$. The important tools for minimizing the severity of infection in children include antiviral therapy, particularly in patients with preexisting neurological disorders or chronic lung disease, and vaccination.

There are some limitations to our study. Virus detection (except for H1N1) was evaluated by IIF, which is not a molecular test with increased sensitivity. The IIF assay can display low sensitivity for ADV and IF detection ${ }^{5}$. Moreover, the lack of data about other respiratory viruses, such as rhinovirus, bocavirus, and metapneumovirus, is a limitation in this study. However, this study profiled important respiratory viruses in the capital of the Brazilian State of Rio Grande do Sul, including data on the H1N1 pandemic.

In summary, each respiratory virus can display a distinct pattern of behavior, causing hospitalization, nosocomial outbreaks, or even mortality in children. Monitoring and surveillance of wellknown respiratory viruses and unexpected emerging threats play an important role in guiding management and minimizing the impact on the community. Consequently, viral surveillance studies are important to guide the timing of prophylaxis and other interventions. Surveillance of RSV peaks, for example, helps track RSV seasonality and thus supports planning of prophylaxis for vulnerable children. Adequate surveillance of influenza plays a major role in the design of appropriate vaccines, the planning of prophylaxis schedules, and the detection of novel viruses. Thus, respiratory illness surveillance strategies can reduce healthcare costs, morbidity, and mortality.

\section{CONFLICT OF INTEREST}

The authors declare that there is no conflict of interest.

\section{FINANCIAL SUPPORT}

Fundo de Incentivo à Pesquisa e Ensino do Hospital de Clínicas de Porto Alegre (FIPE/HCPA), Fundação de Amparo à Pesquisa do Estado do Rio Grande do Sul (FAPERGS) and Conselho Nacional de Desenvolvimento Cientifico e Tecnológico (CNPq).

\section{REFERENCES}

1. World Health Organization/United Nations (WHO/UN) Children's Fund. Global action plan for prevention and control of pneumonia (GAPP). Geneva: World Health Organization and the United Nations Children's Fund, November 2009.

2. Cabello C, Manjarrez M, Olvera R, Villalba J, Valle L, Paramo I. Frequency of viruses associated with acute respiratory infections in children younger than five years of age at a locality of Mexico City. Mem Inst Oswaldo Cruz 2006; 10:21-24.

3. Olofsson S, Brittain-Long R, Andersson LM, Westin J, Lindh M. PCR for detection of respiratory viruses: seasonal variations of virus infections. Expert Rev Anti Infect Ther 2011; 9:615-626.

4. O' Connell K, Boo TW, Keady D, Niriain U, O' Donovan D, Commane M, et al. Use of Palivizumab and infection control measures to control an outbreak of respiratory syncytial virus in neonatal intensive care unit confirmed by real-time polymerase chain reaction. J Hosp Infect 2011; 77:338-342.

5. Pilger DA, Cantarelli VV, Amantea SL, Leistner-Segal S. Detection of human bocavirus and human metapneumovirus by real-time PCR from patients with respiratory symptoms in Southern Brazil. Mem Inst Oswaldo Cruz 2011; 106:56-60.

6. Murray EL, Klein M, Brondi L, McGowan Jr JE, van Mels C, Brooks WA, et al. Rainfall, household crowding, and acute respiratory infections in the tropics. Epidemiol Infect 2012; 140:78-86.

7. World Health Organization. CDC protocol of real time RT-PCR for swine influenza A (H1N1). Atlanta: WHO, 2009.

8. Mlinaric-Galinovic G, Varda-Brkic D. Nosocomial respiratory syncytial virus infections in children's wards. Diagn Microbiol Infect Dis 2000; 37:237-246

9. Tregoning JS, Schwarze J. Respiratory viral infections in infants: causes, clinical symptoms, virology, and immunology. Clin Microbiol Rev 2010; 23:74-98.

10. Weckx LY, Fernandes MM, Monteiro AI, Souza AR, Moraes-Pinto MI. Optimization strategy to minimize wastage of palivizumab during the 2008 RSV season in São Paulo, Brazil. J Trop Pediatr 2009; 55: 341-342.

11. Mak GC, Wong AH, Ho WY, Lim W. The impact of pandemic influenza A (H1N1) 2009 the circulation of respiratory viruses 2009 - 2011. Influenza Other Resp Viruses 2012; 6:6-10.

12. Lynch JP, Fishbein M, Echavarria M. Adenovirus. Semin Respir Crit Care Med 2011; 32:494-511.

13. Libster R, Bugna J, Coviello S, Hijano DR, Dunaiewsky M, Reynoso N, et al. Pediatric hospitalizations associated with 2009 pandemic influenza A (H1N1) in Argentina. N Engl J Med 2010; 362:45-55.

14. Yamaguchi M, Sano Y, Dapat IC, Saito R, Suzuki Y, Kumaki A, et al. High Frequency of Repeated Infections Due to Emerging Genotypes of Human Respiratory Syncytial Viruses among Children during Eight Successive Epidemic Seasons in Japan. J Clin Microbiol 2011; 49:1034-1040.

15. Moretti ML, Sinkoc V, Cardoso LG, Camargo GJ, Bachur LF, Hofling CC, et al. Lessons from the epidemiological surveillance program, during the influenza A (H1N1) virus epidemic, in a reference university hospital of Southeastern Brazil. Rev Soc Bras Med Trop 2011; 44:405-411. 